\title{
Prospects of Diagnostics with Optical Diffraction Radiation in Hadron Colliders
}

\author{
Tanaji Sen, Vic Scarpine and Randy Thurman-Keup, FNAL, Batavia, IL 60510
}

\section{Abstract}

Optical diffraction radiation has been observed and recently used to measure the beam size of electrons at KEK. This non-invasive technique also holds promise for imaging beams close to the interaction point in hadron colliders. In this paper we consider the feasibility of this technique for the Tevatron and the LHC.

\section{INTRODUCTION}

Optical diffraction radiation (ODR) has been successfully used to measure the beam position and beam size in several extraction beam lines [1, 2, 3]. If feasible, this would be a complementary method of measuring important beam parameters in present and future colliders. In this paper we explore the possibility of using ODR from hadron beams in the Tevatron, RHIC and the LHC.

ODR is similar to optical transition radiation (OTR) except that it is emitted when a charged particle moves in the vicinity of a conducting target rather than through the target as is the case with transition radiation. The radiation is emitted in cones both in the forward direction and in the backward direction around the axis of specular reflection. Among the several attractive features of this radiation for beam diagnostics is its non-invasive nature. The backward radiation is emitted at the same longitudinal position as the target if the target is inclined at $45^{\circ}$ to the beam axis. This is an advantage when compared to synchrotron radiation. The radiation is instantaneous and if the intensity is high enough, it can be used for bunch by bunch diagnostics at colliders.

At the simplest level, ODR can be characterized by three basic parameters: the impact parameter $b$ (closeness of the beam to the target), beam energy $(\gamma)$ and wavelength of detected radiation $(\lambda)$. The dimensionless parameter $2 \pi b /(\gamma \lambda)$ should be order one or smaller in order to generate ODR of sufficient intensity. This can be understood at a basic level from the Fourier components of the field of a charged particle. For a particle of charge $e$ moving along the $z$ axis with uniform speed $v$, the Fourier components of the scalar potential describing the field is

$$
\phi_{\omega} \simeq \frac{e}{v \sqrt{2 \pi k r_{\perp} / \gamma}} \exp \left[-\frac{k r_{\perp}}{\gamma}\right] \exp [i k z]
$$

where $r_{\perp}=\sqrt{x^{2}+y^{2}}$ is the transverse distance from the particle and $k=2 \pi / \gamma=\omega / v$. The field is exponentially damped in the transverse direction with an e-folding length of $\gamma \lambda /(2 \pi)$. The target needs to be within an e-folding length of the beam in order to generate a measurable signal but at the same time the target must not be an aperture restriction. The impact parameter $b$ must satisfy dual contstraints : $\kappa=2 \pi b /(\gamma \lambda) \sim 1$ and $b \gg \sigma$ where $\sigma$ is the
RMS beam size. This constrains the usable ODR wavelength.

\section{ODR OBSERVABLES}

Following the proposal [4] to use ODR for beam diagnostics, beam position, beam size and divergence have been measured using one of the following ODR characteristics: near-field and far-field intensity, angular distribution of the far-field intensity, polarization and the frequency spectrum. When using a slit target for example, the ODR intensity is a minimum when the beam is centered in the slit. Beam size has been extracted from the ratio of minimum to maximum in the angular distribution of the far-field intensity.

Measuring the beam position (absolute or relative) close to the interaction point (IP) in a collider is very useful in maximizing the luminosity which requires that both beams are centered on one another. In addition, a beam size measurement in the interaction region is very useful in correcting optics errors in this region and achieving the desired spot size at the IP. Continuous monitoring and correction of these parameters via a non-invasive technique such as ODR is a desirable way of optimizing the luminosity during a collision run.

\section{PARAMETERS FOR HADRON COLLIDERS}

In order to generate the maximum possible diffraction radiation, the wavelength $(\lambda)$ of radiation detected and the slit width $(a)$ need to be carefully chosen. The beam size is determined by the choice of longitudinal location of the target in the ring. Furthermore the target should not impose an aperture restriction, i.e. $a_{\text {eff }}=2 N_{c} \sigma$ where $a_{\text {eff }}$ is the effective slit width and $N_{c}$ is the required dimensionless clearance. In turn the slit width and wavelength are related by the dimensionless parameters $\kappa$ which is required to be of order one. This simple argument requires the wavelength to be chosen as $\lambda=\pi N_{c} \sigma /(\gamma \kappa)$. Table 1 shows the basic parameters of the Tevatron, RHIC and the LHC obtained by setting $N_{c}=12$ and $\kappa=1$. The location in the Tevatron was chosen to be at the pick-off mirror for the synchrotron radiation monitor. The locations in RHIC and LHC were chosen to be in the straight sections of the interaction regions: in RHIC at a distance of $9 \mathrm{~m}$ from the IP and in the LHC at a distance of $18 \mathrm{~m}$ from the IP. These locations are well outside the detector and before the focusing quadrupoles but are otherwise arbitrary. These choices can be refined on subsequent evaluations. This simple evaluation shows the required wavelength to be in the infra-red regime for the Tevatron and LHC while it is in the far infra- 


\begin{tabular}{|l|c|c|c|}
\hline & Tevatron & RHIC & LHC \\
\hline Energy $[\mathrm{TeV}]$ & 0.98 & 0.25 & 7.0 \\
Beta function at target $[\mathrm{m}]$ & 50 & 82 & 1296 \\
Beam size at target $[\mu \mathrm{m}]$ & 399 & 1012 & 807 \\
Target clearance $N_{c}[\sigma]$ & 12 & 12 & 12 \\
Wavelength $[\mu \mathrm{m}]$ & 14.4 & 143 & 4.1 \\
Far-field distance $[\mathrm{m}]$ & 2.5 & 1.6 & 36.1 \\
\hline
\end{tabular}

Table 1: Relevant parameters if the dimensionless parameter $\kappa=1$. For RHIC, the design top energy is chosen while for the LHC, we use the upgrade optics in the IR with $\beta^{*}=0.25 \mathrm{~m}$.

red for RHIC. The far-field distance is the distance beyond which all points on the target appear to be radiating in phase and so-called "pre-wave zone" effects are not significant.

A more accurate evaluation requires use of the intensity spectrum. We use the expression for the differential angular spectrum of the vertical polarization of the intensity for a Gaussian beam taken from Reference [2].

$$
\begin{aligned}
\frac{d^{2} I_{y}}{d \omega d \Omega}= & \frac{\alpha \gamma^{2}}{4 \pi^{2}} \frac{\exp \left[-\kappa \eta_{x}\right]}{\eta_{x}^{2}+t_{y}^{2}}\left\{\operatorname { e x p } \left[\frac{\left.2 \kappa^{2} \sigma_{y}^{2} \eta_{x}^{2}\right)}{a_{\text {eff }}^{2}} \cosh \left[\frac{2 \kappa d_{\text {off }} \eta_{x}}{a_{\text {eff }}}\right]\right.\right. \\
& \left.-\cos \left[\kappa t_{y}+2 \psi\right]\right\}
\end{aligned}
$$

where $\alpha$ is the fine structure constant, $\theta_{x, y}=t_{x, y} / \gamma$ are the observation angles relative to the direction of specular reflection, $\eta_{x}^{2}=1+t_{x}^{2}, a_{e f f}$ is the effective slit width, $d_{o f f}$ is the beam offset from the center of the slit and $\psi=$ $\arctan \left[t_{y} / \eta_{x}\right]$.

We consider the Tevatron in more detail. Figures 1 and 2 show the angular distribution of the intensity projected onto the $\theta_{y}$ axis for wavelengths of $5 \mu \mathrm{m}$ and $10 \mu \mathrm{m}$ respectively. The beam is taken to be centered in the slit in both cases. We see the characteristic features of the minimum at $\theta_{y}=0$ and the nearby peaks. The ratio of the minimum to maximum intensity at $10 \mu \mathrm{m}$ wavelength is about 0.08 this should be within detectable range and therefore usable in order to extract the beam size. The intensity peak at $5 \mu \mathrm{m}$ is about 500 times smaller than at $10 \mu \mathrm{m}$. This reinforces the conclusion that sufficient intensity of ODR in the Tevatron will be generated at wavelengths closer to $10 \mu \mathrm{m}$.

This intensity is also a function of the slit width, increasing as the width decreases. Figure 3 shows the ratio of the minimum to maximum intensity for slit widths in the range of $12-24 \sigma$.

Finally we examine the impact of a beam offset on the intensity. The central minimum shifts away from $\theta_{y}=0$ when the beam is off-centered in the slit. The intensity change at this angle (i.e. along the direction of specular reflection) can be used to measure the beam position. Figure 4 shows the $\%$ change in intensity as the beam offset changes. For example at an offset of $0.1 \sigma$, the intensity changes by about $1 \%$ and increases quadratically to about $27 \%$ for a beam offset of $0.5 \sigma$.

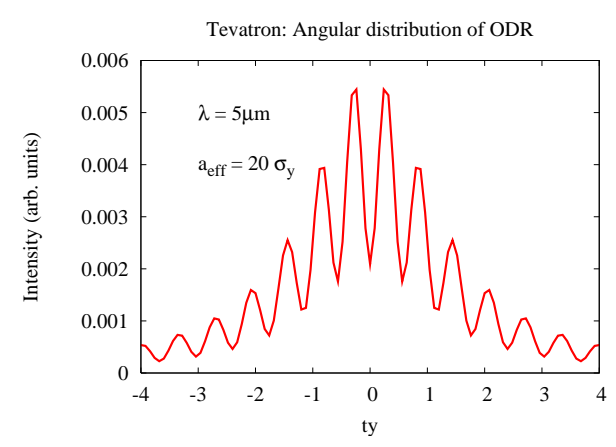

Figure 1: Tevatron: Angular distribution of the ODR intensity vs $t_{y}=\gamma \theta_{y}$ at $5 \mu \mathrm{m}$ wavelength with a $20 \sigma_{y}$ effective slit width.

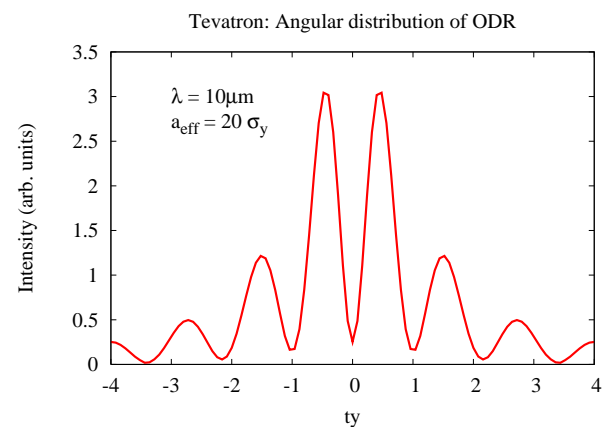

Figure 2: Tevatron: Angular distribution of the ODR intensity vs $t_{y}=\gamma \theta_{y}$ at $10 \mu \mathrm{m}$ wavelength with a $20 \sigma_{y}$ effective slit width.

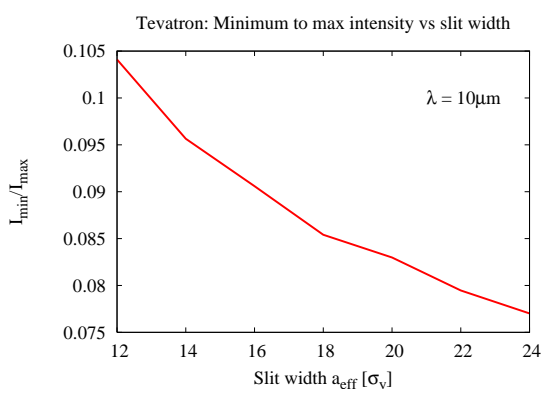

Figure 3: Tevatron: Ratio of minimum to maximum ODR intensity vs the effective slit width at $10 \mu \mathrm{m}$ wavelength..

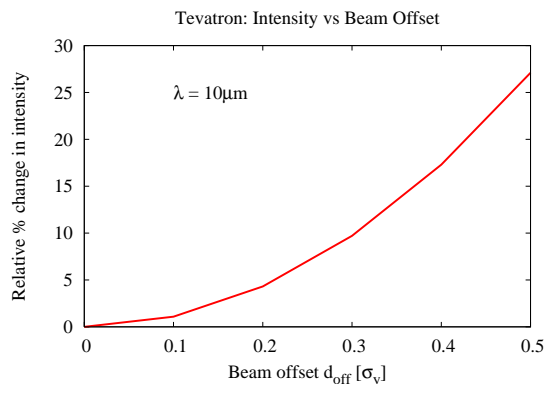

Figure 4: Tevatron: Relative change (\%) in ODR intensity at $\theta_{y}=0$ as a function of the beam offset in units of the rms beam size. 


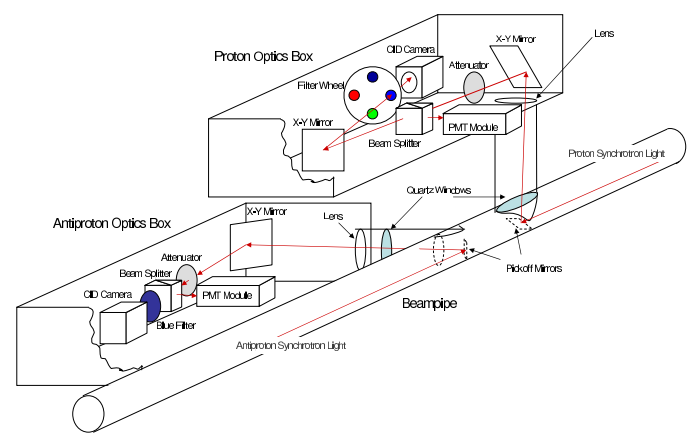

Figure 5: Schematic of the SyncLite monitor in the Tevatron

\section{EXPERIMENTAL ISSUES}

We consider first the possibility of modifying the existing synchrotron radiation monitors in the Tevatron to detect ODR. These monitors (one each for protons and antiprotons) are located in a $3 \mathrm{~m}$ long non-cryogenic section of the ring between two dipole magnets. Each device consists of a movable mirror for intercepting the light originating from the far edge of the adjacent dipole, and a $1 \mathrm{~m}$ long lighttight box housing various optical elements for processing the light, see Figure 5. To use these monitors for ODR detection without permanently disabling the existing functionalities requires a number of modifications. A masking element must be installed just upstream of the proton mirror to block the synchrotron radiation from the mirror. The mirror is designed to be able to move to within $\sim 7 \mathrm{~mm}$ of the center of the beampipe. While the distance to the beam is smaller because of the helical orbits, the target-beam distance is required to be somewhat smaller than $5 \mathrm{~mm}$, see Table 1. Hence the mirror insertion device may need to be modified. The light exits the beampipe through a quartz vacuum window and traverses a $1500 \mathrm{~mm}$ focal length lens which would have to be re-mounted as a flip-in type optical element. At this point the light would have to be redirected by a mirror to either the existing system, or the new ODR system. Because of the changes that are needed to the existing system, there are few advantages to using it. A better approach would be to choose another location. The straight section around $\mathrm{C} 0$ in the Tevatron has available space where an ODR detector could conceivably be installed.

To install a new detector, a new beampipe section with a motorized insertion device would need to be constructed. It could probably contain both the synchrotron radiation masking element and the ODR screen. The light would need to be detected by either a camera device or a single element detector mounted on a 2D movable platform. The detection device would be housed in a light tight box which could be evacuated to avoid water vapor. A camera type device would require a DAQ system to obtain the image, while a single detector element would need an A/D to digitize the signal.

Light detection and image formation in the infrared regime offers it own set of issues. For the LHC, 4 micron radiation is in the near-infrared spectral range ( 0.7 to 5 microns) while for the Tevatron, 14 microns in the midinfrared range (5 to $\sim 25$ microns). Standard optical vacuum windows such as fused quartz have almost no transmission from 3.5 microns to above $\sim 50$ microns. Optical transmission of these infrared wavelengths requires special materials such as diamond. In addition, water vapor absorption starts to strongly reduce infrared transmission. Both issues of vacuum window transmission and water absorption can be eliminated by having the entire optical system in vacuum.

Infrared detection can also be accomplished by using pyroelectric detectors. These devices are broad-band and cheaper but are less sensitive than photoconductive detectors. As an example, DESY has developed a multi-channel coherent radiation spectrometer using pyroelectric detectors to measure the infrared spectrum that can be generated by synchrotron, transition or diffractive radiation [5].

Detection of near and mid infrared radiation requires detectors matched close to their individual wavelengths. In general, these detectors are cryogenically cooled to reduce dark current which is required for any useful operation. Different types of detector materials are used for photoconductive materials such as $\mathrm{InSb}, \mathrm{HgCdTe}, \mathrm{PbS}, \mathrm{PbSe}$ and quantum well infrared photodetectors (QWIP). These materials have been constructed in single element and multielement (camera) type devices. Imaging cameras exist for both near and mid infrared regions. InSb detectors of 640 by 512 pixels are sensitive in the 3 to 5 micron range while similar pixel size QWIP detectors cover the range of 7.5 to 13.5 microns. Single element detectors of these materials can be used to measure the intensity of a portion of the ODR spatial distribution. However, if optical lenses are required to collect and focus the ODR for these infrared detectors then the previously mentioned optical material transmission becomes an issue. This problem may be partially resolved by using gold-coated focusing mirrors for light collection and image formation.

\section{SUMMARY}

Our initial exploration of using ODR for beam diagnostics in hadron colliders shows that sufficient radiation will be generated at wavelengths in the few to several microns range. The issues involved in detecting near and mid infrared radiation need to be studied in greater detail.

\section{REFERENCES}

[1] T. Muto et al,Phys Rev Lett, 90, 104801 (2003)

[2] P. Karataev et al,Phys Rev Lett, 93, 244802 (2004)

[3] A. Lumpkin et al, Phys Rev. ST AB, 10, 022802 (2007)

[4] M. Castellano, Nucl Instr. Mrth. A. 394, 275 (1997)

[5] H. Delsim-Hashemi et al, Proceedings of the 27th International Free Electron Laser Conference, pg 514 (2005) 